\title{
REVISÃO DA QUALIDADE DE ESTUDOS DE IMPACTO AMBIENTAL DE PEQUENAS CENTRAIS HIDRELÉTRICAS
}

\section{QUALITY REVIEW OF ENVIRONMENTAL IMPACT STATEMENTS APPLIED TO SMALL HYDROPOWER PLANTS}

\section{Marcelo Montaño ${ }^{1}$; Alexandre Fernandes Carvalho ${ }^{2}$; Carina Sernaglia Gomes ${ }^{2}$; Carla Natacha Marcolino Polaz ${ }^{3}$; Carolina Oliveira Jordão ${ }^{4}$; Marcelo Pereira de Souza ${ }^{5}$}

\author{
${ }^{1}$ Escola de Engenharia de São Carlos - EESC/USP. E-mail minduim@sc.usp.br \\ ${ }^{2}$ Programa de Pós-Graduação em Ciências da Engenharia Ambiental, Universidade de \\ São Paulo. E-mail alexandref59@ hotmail.com; carinasg@usp.br \\ ${ }^{3}$ Centro Nacional de Pesquisa e Conservação de Peixes Continentais - \\ CEPTA/ICMBio, Pirassununga, SP. E-mail carlapolaz@yahoo.com.br \\ ${ }^{4}$ Instituto Centro de Vida - MS. E-mail jord.usp@gmail.com \\ ${ }^{5}$ Faculdade de Filosofia Ciências e Letras de Ribeirão Preto, Universidade de São \\ Paulo. E-mail mps@usp.br
}

\begin{abstract}
RESUMO
A matriz elétrica brasileira apoia-se sobre empreendimentos hidrelétricos, com evidente predominância de grandes empreendimentos geradores de energia. Nos últimos anos, as Pequenas Centrais Hidrelétricas (PCHs) têm ganhado destaque dentre os empreendimentos que constituem o parque gerador no país, estimuladas pela perspectiva de um menor nível de conflitos socioambientais em comparação com os grandes empreendimentos. Ocorre que, da mesma forma que grandes usinas, as PCHs apresentam uma gama de impactos ambientais que devem ser avaliados previamente à sua implantação. Considerando os procedimentos aplicados atualmente no processo de licenciamento ambiental brasileiro, o presente trabalho tem como objetivo verificar a aplicabilidade de critérios orientadores da avaliação da qualidade técnica de Estudos de Impactos Ambientais de PCHs. A fim de ilustrar as discussões apresentadas, três estudos de impacto elaborados para essa tipologia de empreendimento foram analisados, de modo individual e coletivo. $\mathrm{O}$ conjunto de critérios aplicados mostrou-se adequado para a revisão da qualidade dos estudos apresentados, mostrando-se útil para diminuir a variabilidade e discricionariedade durante a análise técnica dos estudos ambientais que subsidiam o processo de licenciamento ambiental.
\end{abstract}

Palavras-chave: Avaliação de Impacto Ambiental. Licenciamento Ambiental. Efetividade. Instrumentos de Política Ambiental. 


\begin{abstract}
The Brazilian electric mix is based on hydroelectric projects. Among these, the Small Hydropower Plants have gained space: its implementation has been strongly encouraged by medium and longterm government programs. Just as large power plants, the small ones also have a range of environmental impacts. Considering the procedures currently applied in the Brazilian environmental licensing process, the objective of this study was to verify the applicability of a matrix of criteria to support the assessment of technical quality for the Environmental Impacts Studies (EIS) from Small Hydropower Plants. Three case studies were selected, both located in Minas Gerais. The matrix was adequate for the analysis of the studies presented. Meanwhile, the studies analyzed have not clearly demonstrates the environmental viability of the projects. It discusses here the review of environmental licensing for Small Hydropower Plants.
\end{abstract}

Keywords: Environmental Impact Assessment. Environmental Licensing. Effectiveness. Environmental Policy Instruments.

\title{
1. INTRODUÇÃO
}

Em virtude do aumento da demanda de energia elétrica no país, e dada a complexidade encontrada para o seu atendimento, as Pequenas Centrais Hidrelétricas (PCHs) têm sido apontadas como uma importante alternativa para o Brasil. Apoiadas na ideia de rapidez de implantação com baixo impacto ambiental, são ainda fontes de energia renovável, passíveis de obtenção de crédito de carbono, cuja construção, produção e manutenção envolvem processos conhecidos pelos meios técnicos e industriais (MME, 2009). Assim, o governo vem incentivando a construção de PCHs por meio de medidas regulatórias e legais, que convergem para planos e programas de incentivo a exemplo do PROINFA (Programa de Incentivo às Fontes Alternativas de Energia Elétrica), do PDE (Plano Decenal de Energia) (CERPCH, 2006) e, mais recentemente, do PAC (Programa de Aceleração do Crescimento).

O histórico de conflitos relacionados aos impactos ambientais provocados por grandes empreendimentos hidrelétricos tem contribuído para reforçar a opção pela implantação de PCHs, especialmente porque esta tipologia de atividade encontra um caminho menos exigente, em relação aos grandes aproveitamentos hidrelétricos, em termos dos procedimentos aplicados ao licenciamento ambiental.

Ainda que possam ser consideradas uma opção para geração de energia elétrica de menor potencial impactante quando comparadas com outras alternativas para produção de energia em larga escala (THIAGO FILHO et al., 2003), as PCHs não devem ser negligenciadas em relação aos seus impactos. A esse respeito, Nascimento e Drummond (2003) afirmaram ser discutível a adoção do modelo de implantação de PCHs sem a avaliação de seus efeitos cumulativos.

Dentre os principais impactos provocados pela implantação de PCHs destacam-se: alteração do regime de escoamento dos corpos de água, perda de vegetação e desmatamento, eutrofização, alteração da morfologia de rios, modificação da paisagem, deslocamento de população e outros. Esses mesmos impactos, em menor magnitude, podem ser verificados nos empreendimentos hidrelétricos de maior porte (BARBOSA e DUPAS, 2006; TSOUTSOS et al., 2007). Além disso, devem ser levados em consideração o seu potencial de causar impactos cumulativos a partir da implantação de várias PCHs operando em uma mesma bacia (PINHO et al., 2007).

A resolução CONAMA 01/86 (BRASIL, 1986) fornece, em seu Art. $2^{\circ}$., uma lista positiva de atividades potencialmente impactantes do meio e, portanto, passíveis de EIA/RIMA: usinas de geração de eletricidade, qualquer que seja a fonte primária de energia, com potência acima de 10 MW, são mencionadas no inciso XI. Por força dessa regulamentação, as PCHs têm sido frequentemente avaliadas com base nesta modalidade de estudo. 
É importante destacar que o EIA - independentemente da tipologia de atividade retratada deve discutir as condições para a viabilidade ambiental dos empreendimentos, a partir da avaliação de seus impactos (MONTAÑO e SOUZA, 2008), o que torna a análise técnica dos estudos ambientais uma das etapas fundamentais da Avaliação de Impacto Ambiental. Afinal, é a partir desta etapa que as informações relevantes para a tomada de decisão serão disponibilizadas aos diferentes grupos de interesse, levando-se em conta a interpretação dos órgãos e agentes técnicos envolvidos.

Conforme as considerações de Sánchez (2006) o alto tempo de tramitação dos processos de concessão das licenças ambientais para aproveitamentos hidrelétricos relaciona-se, dentre outros, com a qualidade dos estudos ambientais e a inerente dificuldade para análise e posterior elaboração de pareceres técnicos. A revisão dos estudos ambientais por parte dos órgãos licenciadores, quando respaldada por procedimentos sistemáticos de análise e interpretação, pode contribuir para a aprendizagem e aperfeiçoamento do sistema de AIA (Avaliação de Impacto Ambiental) de modo geral.

Corroborando esta colocação, Barker e Wood (1999) aplicaram o método originalmente proposto por Lee e Colley (1992) em 24 estudos ambientais provenientes de 8 países europeus (Bélgica, Dinamarca, Grécia, Irlanda, Portugal, Alemanha, Espanha e Reino Unido), tendo confirmado como benéfico a indução de menor variabilidade na interpretação das informações entre os analistas.

Ao refletirem sobre a relevância de cada um dos critérios para revisão da qualidade de estudos ambientais, Pinho et al. (2007) fizeram destaque ao estudo de alternativas: tendo em vista a natureza dos empreendimentos em análise, a decisão sobre o tipo, a potência e a localização do empreendimentos é um processo complexo, e que, portanto deveria em essência contemplar alternativas não somente tecnológicas, mas sobretudo locacionais. De acordo com os mesmos autores, as principais deficiências são: nenhuma ou poucas alternativas são consideradas nos estudos; quando existem, elas são descritas sumariamente, sem a quantificação dos devidos impactos; a justificativa para a alternativa escolhida não é apresentada e, finalmente, alternativas locacionais raramente são contempladas.

Montaño e Souza (2008) argumentam que não há análise de viabilidade ambiental sem a consideração específica do aspecto locacional, cuja discussão ocorre ao longo da fase de licenciamento prévio. No caso brasileiro, um estudo qualitativo conduzido pelo Ministério Público da União acerca das deficiências de EIAs aponta que a ausência de proposição de alternativas, a apresentação de alternativas reconhecidamente inferiores à selecionada no EIA e a prevalência dos aspectos econômicos sobre os ambientais na escolha das alternativas estão entre os principais problemas relacionados aos estudos de alternativas tecnológicas e locacionais dentro dos EIAs (MINISTÉRIO PÚBLICO DA UNIÃO, 2004).

Glasson e Salvador (2000) se debruçaram sobre os problemas relacionados à avaliação de impactos ambientais no Brasil e, consequentemente, a todo processo de licenciamento ambiental de empreendimentos. Segundo esses autores, um dos gargalos da AIA no país é a morosidade para a análise e emissão das licenças. Em âmbito nacional e internacional, o licenciamento ambiental brasileiro é traduzido como um mecanismo administrativo excessivamente burocrático (BANCO MUNDIAL, 2008). Esse atraso nos procedimentos faz com que os EIAs, que deveriam fornecer os subsídios técnicos para a tomada de decisão sobre a licença prévia (LP), que define localização, sejam considerados somente à frente, já na fase de emissão da licença de instalação (LI). Dessa maneira, o papel preventivo do EIA enquanto instrumento de planejamento fica severamente comprometido.

Com relação aos programas de monitoramento, embora amparados pela legislação, na prática, não existem ou são raros (GLASSON e SALVADOR, 2000). A participação pública, assunto abordado também por Pinho et al. (2007), possui abrangência limitada; acrescenta-se o fato 
de que o RIMA, na maioria dos casos, não está em linguagem acessível, o que restringe ainda mais as oportunidades de envolvimento da sociedade.

Essas constatações servem ainda para corroborar o trabalho de Hickie e Wade (1998). Ao investigarem 14 estudos ambientais no Reino Unido, encontraram as mesmas deficiências: problemas na comunicação dos resultados, bases de referência inadequadas, análise superficial de impactos, falhas na abordagem de alternativas, falta de mecanismos que assegurem a efetiva implementação das medidas mitigadoras propostas, bem como dos programas de monitoramento.

Em se tratando de instrumentos para a revisão da qualidade de EIAs, destacam-se aqueles elaborados a partir de listagens de verificação, ferramentas relativamente simples e que têm a vantagem de poder ser utilizadas por diferentes interessados sem grandes desvios de interpretação (SÁNCHEZ, 2006).

A Comissão Europeia disponibiliza uma guia metodológica para revisão da qualidade dos estudos ambientais (EUROPEAN COMMISSION, 2001) para orientação dos países-membros da UE. As aplicações efetuadas por Peterson (2010) em 50 estudos de impacto ambiental finalizados entre 2001 e 2005 na Estônia, e Canelas et al. (2005) com 46 estudos de impacto ambiental elaborados entre 1998 e 2003 em Portugal e Espanha, ilustram a aplicabilidade deste método.

Diversas adaptações para os guias mencionados têm sido elaboradas em diferentes contextos, focando em duas situações específicas: adaptações referentes aos critérios de análise da qualidade dos EIAs, e adaptações para aplicações em tipologias específicas de empreendimentos.

Com relação ao primeiro grupo, Glasson et al. (2005) apresentaram uma adaptação para o método desenvolvido por Lee e Coley (1992), organizando os critérios de análise em quatro seções: descrição do empreendimento, do meio e condições de diagnóstico; identificação e avaliação de impactos; alternativas e mitigação; e comunicação dos resultados.

Já para as adaptações relacionadas ao segundo grupo encontram-se, por exemplo, o guia proposto por Sánchez (2006) para aplicações em estudos de impacto de rodovias e o guia proposto por Pinho et al. (2007) voltado para pequenas centrais hidrelétricas e aplicado a 13 estudos de impacto de PCHs em Portugal. Em ambos, destaca-se a existência de uma série de critérios de análise voltados para determinadas características dos projetos a que se referem, refletindo a necessidade de grande conhecimento destas tipologias por parte de seus propositores.

Diante deste contexto de expansão das PCHs, é importante que os órgãos ambientais tenham à sua disposição instrumentos que lhes auxiliem no processo de revisão de relatórios e estudos de impacto que instruem os pedidos de licença ambiental. Além disso, a aplicação de um conjunto de critérios especificamente estabelecidos para a revisão sistemática dos estudos de impacto oferece aos tomadores de decisão a possibilidade de reduzir a subjetividade e a discricionariedade nas avaliações das solicitações de licença. Um efeito decorrente, discutido na literatura, remete aos benefícios para os operadores dos sistemas de Avaliação de Impacto Ambiental (AIA) derivados do ganho de eficiência que se obtém com a aprendizagem induzida pela sistematização de critérios para revisão da qualidade dos estudos de impacto.

Sendo assim, o presente trabalho discute a aplicabilidade de um conjunto de critérios para revisão de estudos de impacto de PCHs elaborados em processos de AIA. Para tanto, avaliou-se a qualidade técnica de três EIA/RIMAs aplicados a esta tipologia de atividade discutindo-se as potenciais contribuições para o processo de tomada de decisão.

\section{MATERIAL E MÉTODOS}

Os procedimentos metodológicos propostos por Pinho et al. (2007) foram aplicados a três estudos de impacto de PCHs localizadas nos estados de Minas Gerais, Paraná e São Paulo, tendo sido selecionados aleatoriamente e posteriormente triados em função da possibilidade de acesso ao 
conteúdo integral de sua documentação (textos, mapas, projeto básico, estudos específicos, complementações, anexos, entre outros). A Tabela 1, a seguir, apresenta algumas características para os empreendimentos selecionados, denominados aqui de PCH1 a PCH3.

Tabela 1 - Características dos empreendimentos selecionados para análise

\begin{tabular}{|c|c|c|c|}
\hline \multirow{2}{*}{ Características } & \multicolumn{3}{|c|}{ Empreendimentos } \\
\cline { 2 - 4 } potência instalada (MW) & PCH1 & PCH2 & PCH3 \\
\hline energia firme (MW) & 22,5 & 14 & 27 \\
\hline área ocupada (ha) & 800 & não consta & 15 \\
\hline área inundada (ha) & 580 & 232 & 960 \\
\hline obtenção da LP & 2007 & 100 & 399 \\
\hline entrada em operação & 2013 & não consta & não consta \\
\hline órgão licenciador & $\begin{array}{c}\text { SUPRAM/LM } \\
\text { (MG) }\end{array}$ & $\begin{array}{c}\text { SUPRAM/LM } \\
\text { (MG) }\end{array}$ & IAP (PR) \\
\hline
\end{tabular}

\subsection{Caracterização do objeto de estudo}

Os procedimentos metodológicos empregados na elaboração do presente trabalho envolveram inicialmente a caracterização do conjunto de estudos de impacto analisados, em relação aos argumentos apresentados para a avaliação dos impactos ambientais, a fim de minimizar a ocorrência de algum viés na ponderação dos critérios de avaliação.

Deste modo, os estudos de impacto ambiental foram analisados individualmente e em seguida discutidos coletivamente por um grupo de especialistas em AIA com formação em diferentes áreas. Os pontos discordantes, uma vez identificados por alguma diferença significativa na avaliação de um determinado critério, eram discutidos coletivamente procurando-se um entendimento comum. Para fins de estabelecimento do valor final, a ponderação indicada para cada subcritério reflete a média para as avaliações de cada componente do grupo.

Conforme consta dos EIAs elaborados para as PCHs 1 e 3, foram consideradas as variáveis ambientais indicadoras de dois sistemas principais que se interrelacionam: o sistema representado pelo projeto de engenharia e o sistema ambiental, representado pelas características de sua área de inserção (meios físico, biótico e antrópico). Os estudos foram realizados utilizando-se bibliografia especializada, levantamentos de campo e análise de informações cartográficas, envolvendo a região de implantação do empreendimento. Para o diagnóstico ambiental foram definidas e delimitadas duas unidades espaciais de análise: Área de Influência Direta (AID) e Área de Influência Indireta (AII). Medidas mitigadoras e diversos programas de monitoramento foram propostos.

Em relação à $\mathrm{PCH} 2$, o EIA foi elaborado de acordo com o diagnóstico e a identificação e análise dos impactos para três categorias de área. Foram definidas três unidades espaciais de análise: Área de Influência (AI), Área de Entorno (AE) e Área Diretamente Afetada (ADA), sendo realizadas para cada uma dessas áreas a caracterização, referente ao diagnóstico, a identificação dos impactos e a proposição de medidas mitigadoras e compensatórias.

Para as PCHs 1 e 2, elaboradas segundo as orientações do mesmo órgão ambiental, a avaliação do meio físico foi realizada considerando os seguintes aspectos: clima; hidrografia (caracterização dos cursos de água); geologia; aspectos geotécnicos e geomorfológicos; patrimônio natural; pedologia; aptidão do uso e potencial agrícola dos solos. O meio biótico, por sua vez, foi avaliado considerando os principais grupos indicadores da qualidade ambiental, bem como a avaliação do uso e ocupação do solo. Para os ecossistemas terrestres foram considerados: cobertura vegetal; herpetofauna; avifauna e mastofauna. Para os ecossistemas aquáticos considerou-se: avaliação da qualidade físico-química da água; parâmetros bacteriológicos; fitoplâncton; 
zooplâncton; zoobênton; e ictiofauna. Por fim, o meio antrópico foi avaliado de acordo com os seguintes temas: patrimônio cultural (histórico e arqueológico) e socioeconomia.

No caso da PCH 3, o meio físico foi descrito a partir de variáveis para clima, geologia, geomorfologia, pedologia, hidrografia e hidrosedimentologia. O meio biótico foi descrito de modo mais abrangente a partir dos ecossistemas terrestres e aquáticos, com ênfase para os grupos de flora e fauna, utilizando-se basicamente os mesmos indicadores empregados para as demais PCHs. Finalmente, o meio socioeconômico foi abordado a partir de aspectos da socioeconomia, populações indígenas e quilombolas, patrimônio histórico, cultural e ambiental.

Em relação ao detalhamento das metodologias utilizadas para a avaliação de cada tema, houve diferenças em relação aos EIAs estudados. Para o caso da PCH1, o detalhamento das metodologias foi descrito em capítulo específico. Na etapa de avaliação propriamente dita, foram identificados e analisados os principais impactos ambientais positivos e negativos gerados pelo empreendimento, em suas fases de planejamento, implantação e operação, bem como os principais grupos de interesse a serem afetados por esses impactos. Não foram relacionados, entretanto, os impactos advindos da fase de desativação do empreendimento. No EIA apresentado para a PCH2, as metodologias de análise foram apresentadas no decorrer do documento, conforme os temas tratados. Foram avaliados os impactos positivos e negativos somente das fases da construção e operação do empreendimento, com ênfase aos impactos socioeconômicos. Para a PCH3 foram trabalhadas em separado as ações do empreendimento, componentes ambientais sujeitos ao impacto, e avaliação dos impactos (com descrição das metodologias). Foram avaliados os impactos positivos e negativos para as etapas de planejamento, construção e operação do empreendimento.

Quanto às etapas referentes ao diagnóstico, avaliação dos impactos, prognósticos (referentes à avaliação dos cenários existentes frente às alternativas e, também, frente à consideração de não execução do projeto) e proposição de medidas mitigadoras e compensatórias, para a PCH1, de posse do diagnóstico e da avaliação dos impactos, foram especificadas as ações preventivas e corretivas destinadas à mitigação destes (medidas de minimização, reabilitação e compensação ambiental). Com base no cronograma geral de implantação do projeto, as medidas indicadas foram discutidas por fator ambiental a que se destinam, sendo indicados os responsáveis por sua adoção. Finalmente, foi elaborado um prognóstico da qualidade ambiental futura, considerando-se o meio ambiente como um todo, a partir de duas situações, com e sem a presença do empreendimento.

Para a PCH2, ao contrário, o diagnóstico, a identificação e análise dos impactos foram realizados apenas para uma alternativa locacional. As medidas de compensação e mitigação foram apresentadas de acordo com os temas estudados, meios físico, biológico e antrópico, e o único prognóstico apresentado refere-se à alteração das características socioeconômicas da região, tendo sido apresentadas essas características com e sem a presença do empreendimento, porém, isso não foi realizado para os aspectos referentes ao meio físico e biológico.

Por sua vez, o EIA para a PCH3 também apresentou estudos apenas para uma única alternativa locacional, mantendo o foco das avaliações e prognósticos para cada tema estudado meios físico, biótico e socioeconômico. Ao final, apresenta o que denomina de Análise Integrada, tendo em vista a caracterização do empreendimento de modo global em relação ao cenário inicial (sem o empreendimento) e futuro.

Em relação à última etapa do processo de elaboração do EIA, referente à elaboração do RIMA, previsto pela CONAMA 01/86, para as PCHs 1 e 3 foram apresentados de forma sintética com os resultados finais do EIA e disponibilizados à comunidade interessada em documento à parte. Já o RIMA da PCH2 foi apresentado como sendo praticamente o mesmo documento que o EIA, ou seja, contrariamente ao que preconiza a resolução CONAMA 001/1986. 


\subsection{Procedimentos para revisão da qualidade de EIAs e interpretação dos resultados}

Os critérios propostos por Pinho et al. (2007), dispostos na Tabela 2, abordam 12 seções associadas ao estudo de impacto ambiental: (1) Escopo; (2) Caracterização do projeto; (3) Inclusão de alternativas; (4) Diagnóstico ambiental; (5) Identificação de impactos; (6) Descrição dos impactos previstos; (7) Medidas mitigadoras; (8) Monitoramento; (9) Dificuldades técnicas e ausência de informações; (10) Participação social; (11) Apresentação dos resultados; e (12) RIMA.

A aplicação dos critérios se dá a partir da análise de seu elemento equivalente que integra o EIA: se o elemento não for considerado no EIA será automaticamente atribuído valor zero; se for considerado, ele será avaliado em uma escala com três faixas: Baixo - quando o elemento em questão for considerado de forma incompleta ou tecnicamente insatisfatória; Médio - quando o elemento em questão for considerado de forma razoável ou apenas satisfatória; e Alto - quando for considerado de forma completa e muito satisfatória.

A escala empregada para a revisão da qualidade dos EIAs varia de 0.0 a 3.0, não havendo diferenciação de pesos na ponderação dos critérios e subcritérios. Os resultados numéricos são ainda agrupados em seis categorias qualitativas, que necessariamente refletem as três faixas de qualidade descritas anteriormente: I. Muito fraco $(0.0-0.4)$, quando a maioria dos subcritérios (associados a cada critério) não foi contemplada e os demais o foram de maneira deficiente; II. Fraco (0.5 - 0.9), quando uma quantidade significativa de subcritérios não foi contemplada e alguns outros apresentam deficiências; III. Regular (1.0 - 1.4), quando parte dos subcritérios foi contemplada, mas outra parte permaneceu abaixo de padrões satisfatórios; IV. Satisfatório (1.5 1.9), quando apenas uma pequena parte dos subcritérios não foi contemplada, mas outra parte significativa atendeu níveis satisfatórios; V. Bom $(2.0-2.4)$, quando todos os subcritérios foram contemplados, e a maioria deles em um nível satisfatório; e VI. Muito bom (2.5 - 3.0), quando todos os subcritérios foram contemplados num nível satisfatório.

Tendo em vista que o método de Pinho et al. (2007) não preconiza a aplicação de pesos diferenciados para a revisão dos EIAs, a qualidade geral dos estudos de impacto foi discutida no presente trabalho a partir de parâmetros derivados da média calculada para o conjunto de critérios aplicados. Além disso, para auxiliar na interpretação dos resultados, foram também calculados a mediana e os valores para o $1^{\circ}$ e $3^{\circ}$ quartis para cada estudo de impacto analisado.

Tabela 2 - Critérios e subcritérios adotados para avaliação da qualidade de estudos ambientais de pequenas centrais hidrelétricas. Fonte: Adaptado de Pinho et al. (2007)

\begin{tabular}{|c|l|}
\hline \multirow{4}{*}{ CRITÉRIOS } & \multicolumn{1}{c|}{ SUBCRITÉRIOS } \\
\hline \multirow{4}{*}{ (1) “Scoping" } & (1.1) Identifica os principais temas a serem analisados no relatório do EIA. \\
\cline { 2 - 3 } & $\begin{array}{l}\text { (1.2) Identifica adequadamente os impactos mais significativos. } \\
\text { (1.3) Envolve os principais atores no escopo do EIA. }\end{array}$ \\
\cline { 2 - 3 } (1.4) Descreve e justifica o escopo bem como os métodos utilizados. \\
\hline \multirow{4}{*}{$\begin{array}{l}\text { (2).1) Identifica e caracteriza os diferentes componentes do projeto. } \\
\text { do projeto }\end{array}$} & $\begin{array}{l}\text { (2.2) Caracteriza as fases de construção, operação e desativação; descreve para } \\
\text { cada fase os materiais, a energia utilizada e produzida, os efluentes, resíduos e } \\
\text { emissões produzidos. }\end{array}$ \\
\cline { 2 - 3 } $\begin{array}{l}\text { (2.3) Descreve a relação entre o projeto em âmbito local, regional e nacional que } \\
\text { afetam o meio ambiente circunvizinho. Diagramas, mapas e planos são utilizados } \\
\text { com este objetivo. }\end{array}$ \\
\cline { 2 - 3 } & $\begin{array}{l}\text { (2.4) Descreve a localização do projeto, os seus diferentes acessos e identifica as } \\
\text { áreas sensíveis que poderão ser mais afetadas. }\end{array}$ \\
\cline { 2 - 3 } (2.5) Inclui as razões pelas quais o projeto proposto foi escolhido. \\
\cline { 2 - 3 } & (2.6) Descreve e explica os objetivos do projeto. \\
\hline (3) Inclusão de & (3.1) Apresenta uma avaliação objetiva de todas as alternativas razoáveis. Descreve \\
\hline
\end{tabular}




\begin{tabular}{|c|c|}
\hline \multirow[t]{3}{*}{ alternativas } & $\begin{array}{l}\text { as várias alternativas em relação à localização do projeto, componentes e processos } \\
\text { tecnológicos disponíveis. }\end{array}$ \\
\hline & (3.2) Descreve a alternativa zero, ou seja, a opção de não implantação do projeto. \\
\hline & $\begin{array}{l}\text { (3.3) Explica as razões pelas quais algumas alternativas foram descartadas do } \\
\text { estudo. }\end{array}$ \\
\hline \multirow{4}{*}{$\begin{array}{l}\text { (4) Diagnóstico } \\
\text { ambiental }\end{array}$} & $\begin{array}{l}\text { (4.1) Caracteriza a situação ambiental que deve envolver a descrição e } \\
\text { caracterização de todos os fatores ambientais suscetíveis de serem afetados. }\end{array}$ \\
\hline & $\begin{array}{l}\text { (4.2) Descreve a metodologia para caracterizar cada fator ambiental e os métodos } \\
\text { usados para realizar todos os estudos analíticos. }\end{array}$ \\
\hline & $\begin{array}{l}\text { (4.3) Inclui informação quantitativa sempre que necessária e bases cartográficas } \\
\text { para a melhor compreensão/interpretação dos dados; apresenta justificativas } \\
\text { científicas, sempre que necessário. }\end{array}$ \\
\hline & $\begin{array}{l}\text { (4.4) Inclui a caracterização do patrimônio histórico e arqueológico e todos os } \\
\text { aspectos socioeconômicos relevantes. }\end{array}$ \\
\hline \multirow[b]{2}{*}{$\begin{array}{l}\text { (5) Identificação de } \\
\text { impactos }\end{array}$} & $\begin{array}{l}\text { (5.1) Identifica as principais ações capazes de gerar impactos em todas as fases do } \\
\text { projeto, e aquelas que podem alterar processos naturais. }\end{array}$ \\
\hline & $\begin{array}{l}\text { (5.2) Identifica os impactos do projeto para cada e qualquer fator ambiental } \\
\text { selecionado, nas diferentes fases projeto, utilizando os dados fornecidos pela } \\
\text { pesquisa e análise das condições da área e das características do projeto. }\end{array}$ \\
\hline \multirow{5}{*}{$\begin{array}{l}\text { (6) Descrição dos } \\
\text { impactos previstos }\end{array}$} & $\begin{array}{l}\text { (6.1) Apresenta a metodologia para a predição de impacto e avaliação científica e } \\
\text { indica a incerteza associada a cada prediçáo; os métodos utilizados devem ser } \\
\text { explicados e as razões para escolha do nível de detalhe da análise devem ser } \\
\text { fornecidas. }\end{array}$ \\
\hline & $\begin{array}{l}\text { (6.2) Identifica outros impactos que possam ocorrer após a implementação das } \\
\text { medidas de minimização, e avalia os impactos que possam ocorrer devido às } \\
\text { condições anormais. }\end{array}$ \\
\hline & $\begin{array}{l}\text { (6.3) Evita a introdução de julgamentos subjetivos de valor e a imposição da } \\
\text { opinião dos atores envolvidos sobre a importância dos impactos ambientais ou } \\
\text { sociais. }\end{array}$ \\
\hline & $\begin{array}{l}\text { (6.4) Avalia os impactos de acordo com sua importância relativa, isto é, em relação } \\
\text { às características do impacto tais como a área de influência, o tipo, a natureza, } \\
\text { magnitude, duração, reversibilidade e probabilidade de ocorrência. }\end{array}$ \\
\hline & $\begin{array}{l}\text { (6.5) Caracteriza as relações de causa-efeito de cada impacto ambiental; avaliação } \\
\text { retroativa, impactos cumulativos e sinérgicos. }\end{array}$ \\
\hline \multirow{4}{*}{$\begin{array}{l}\text { (7) Medidas } \\
\text { mitigadoras }\end{array}$} & $\begin{array}{l}\text { (7.1) Descreve as medidas de mitigação ou compensação propostas, com } \\
\text { informações relativas à sua aplicação e eficácia. }\end{array}$ \\
\hline & $\begin{array}{l}\text { (7.2) Fornece evidências do empenho do proponente e sua capacidade para } \\
\text { implementar as medidas mitigadoras. }\end{array}$ \\
\hline & $\begin{array}{l}\text { (7.3) Justifica, quando for o caso, a ausência de propostas de mitigação para os } \\
\text { impactos significativos previamente identificados. }\end{array}$ \\
\hline & $\begin{array}{l}\text { (7.4) Propõe um programa de monitoramento sempre que existir incerteza sobre os } \\
\text { resultados práticos de uma medida mitigadora, permitindo ajustes futuros, se } \\
\text { necessário. }\end{array}$ \\
\hline \multirow{5}{*}{ (8) Monitoramento } & $\begin{array}{l}\text { (8.1) Descreve os objetivos do programa de monitoramentos e sua justificativa } \\
\text { científica. }\end{array}$ \\
\hline & $\begin{array}{l}\text { (8.2) Descreve com precisão quais tipos de indicadores devem ser monitorados, } \\
\text { como e quando devem ser monitorados e que organização/instituição deve ser } \\
\text { responsável pela fiscalização. }\end{array}$ \\
\hline & $\begin{array}{l}\text { (8.3) Fornece evidências do compromisso dos proponentes com o(s) programa(s) } \\
\text { de monitoramento. }\end{array}$ \\
\hline & $\begin{array}{l}\text { (8.4) Identifica outros agentes envolvidos e os papéis específicos desses agentes, se } \\
\text { aplicável. }\end{array}$ \\
\hline & ento para touos os \\
\hline
\end{tabular}




\begin{tabular}{|c|c|}
\hline & ambientais. \\
\hline $\begin{array}{l}\text { (9) Dificuldades } \\
\text { técnicas e ausência } \\
\text { de informacões }\end{array}$ & $\begin{array}{l}\text { (9.1) Identifica as limitações e dificuldades enfrentadas pela equipe técnica durante } \\
\text { a preparação do relatório de EIA. }\end{array}$ \\
\hline \multirow{4}{*}{$\begin{array}{l}\text { (10) Participação } \\
\text { social }\end{array}$} & (10.1) Identifica os grupos de interesse e o público em geral envolvido na consulta. \\
\hline & $\begin{array}{l}\text { (10.2) Incorpora a multiplicidade de valores fornecidos pelo público, especialistas } \\
\text { e grupos de interesse; descreve as ações que foram tomadas para determinar a } \\
\text { opinião das populações locais em relação às consequências sociais dos impactos } \\
\text { previstos. Fornece evidência de consideração das percepções e opiniões da } \\
\text { comunidade. }\end{array}$ \\
\hline & $\begin{array}{l}\text { (10.3) Fornece razões explícitas do porquê certas sugestões não foram } \\
\text { incorporadas ao EIA. }\end{array}$ \\
\hline & (10.4) Identifica o grau de preocupação ou de conflito com o público. \\
\hline \multirow{5}{*}{$\begin{array}{l}\text { (11) Apresentação } \\
\text { dos resultados }\end{array}$} & $\begin{array}{l}\text { (11.1) Apresenta um estudo claro, coerentemente organizado, bem escrito e } \\
\text { facilmente compreensível por não-especialistas e pelo público; fontes de } \\
\text { informação são mencionadas de forma clara; qualquer informação, dados ou } \\
\text { abordagem provenientes de fontes externas é devidamente apoiado por uma } \\
\text { referência; inclui uma lista completa de referências. }\end{array}$ \\
\hline & $\begin{array}{l}\text { (11.2) Indica que grupo de disciplinas técnicas e científicas tem orientado a } \\
\text { preparação do estudo; informa que a legislação/regulamentação foi atendida } \\
\text { adequadamente. }\end{array}$ \\
\hline & $\begin{array}{l}\text { (11.3) Termos técnicos são explicados detalhadamente no glossário ou em texto; os } \\
\text { pareceres e relatórios dos peritos são complementados com uma interpretação } \\
\text { adequada e não apenas anexados ao estudo. }\end{array}$ \\
\hline & (11.4) Indica os nomes e qualificação da equipe técnica. \\
\hline & $\begin{array}{l}\text { (11.5) Apresenta as conclusões finais sobre os impactos positivos e negativos da } \\
\text { proposta, considerando as diferentes fases do projeto. }\end{array}$ \\
\hline \multirow{4}{*}{ (12) RIMA } & $\begin{array}{l}\text { (12.1) Inclui um resumo adequado não técnico (RIMA), que descreve a } \\
\text { constituição geral do projeto e brevemente caracteriza a construção, operação e } \\
\text { desativação. }\end{array}$ \\
\hline & $\begin{array}{l}\text { (12.2) Identifica os principais fatores ambientais a serem afetados pelo projeto, } \\
\text { suas alternativas, seus principais impactos e as propostas de } \\
\text { mitigação/compensação. }\end{array}$ \\
\hline & $\begin{array}{l}\text { (12.3) É claro, conciso e bem escrito, sem excessos ou linguagem técnica ou } \\
\text { estatísticas complexa. Reflete o conteúdo geral do EIA e apresenta as suas } \\
\text { principais conclusões, suas alternativas e medidas mitigadoras e como serão } \\
\text { alcançadas. }\end{array}$ \\
\hline & (12.4) Indica os planos de monitoramento propostos no EIA. \\
\hline
\end{tabular}

\section{RESULTADOS E DISCUSSÃO}

Os resultados obtidos com a aplicação da matriz de critérios empregada no presente trabalho são apresentados na Tabela 3 e ilustrados nas Figuras 1 e 2. 
Tabela 3 - Resultados da aplicação dos critérios e subcritérios para os EIAs analisados, segundo Pinho et al. (2007)

\begin{tabular}{|l|c|c|c|}
\hline \multicolumn{1}{|c|}{ CRITÉRIOS } & PCH1 & PCH2 & PCH3 \\
\hline (1) Escopo & Regular (1.0) & Satisfatório (1.9) & Fraco (0.9) \\
\hline (2) Caracterização do projeto & Satisfatório (1.5) & Satisfatório (1.5) & Bom (2.0) \\
\hline (3) Inclusão de alternativas & Satisfatório (1.7) & Fraco (0.5) & Fraco (0.6) \\
\hline (4) Diagnóstico ambiental & Bom (2.2) & Satisfatório (1.9) & Bom (2.0) \\
\hline (5) Identificação de impactos & Satisfatório (1.5) & Satisfatório (1.5) & Regular (1.4) \\
\hline $\begin{array}{l}\text { (6) Descrição dos impactos } \\
\text { previstos }\end{array}$ & Regular (1.1) & Regular (1.1) & Satisfatório (1.6) \\
\hline (7) Medidas mitigadoras & Regular (1.1) & Regular (1.0) & Regular (1.0) \\
\hline (8) Monitoramento & Satisfatório (1.9) & Satisfatório (1.5) & Regular (1.1) \\
\hline $\begin{array}{l}\text { (9) Dificuldades técnicas e } \\
\text { ausência de informações }\end{array}$ & Regular (1.0) & Regular (1.0) & Fraco (0.8) \\
\hline (10) Participação social & Fraco (0.5) & \multicolumn{1}{|c|}{ Bom (2.0) } & Muito Fraco (0.3) \\
\hline (11) Apresentação dos resultados & Satisfatório (1.8) & Regular (1.3) & Bom (2.0) \\
\hline (12) RIMA & Regular (1.1) & Muito Fraco (0.1) & Regular (1.3) \\
\hline Média & $\mathbf{1 . 3 7}$ & $\mathbf{1 . 2 8}$ & $\mathbf{1 . 2 5}$ \\
\hline Mediana & $\mathbf{1 . 3}$ & $\mathbf{1 . 4}$ & $\mathbf{1 . 2}$ \\
\hline $\mathbf{1}^{\mathbf{0}}$ Quartil & $\mathbf{1 . 0 7 5}$ & $\mathbf{1}$ & $\mathbf{0 . 8 7 5}$ \\
\hline $\mathbf{3}^{\mathbf{0}}$ Quartil & $\mathbf{1 . 7 2 5}$ & $\mathbf{1 . 6}$ & $\mathbf{1 . 7}$ \\
\hline
\end{tabular}

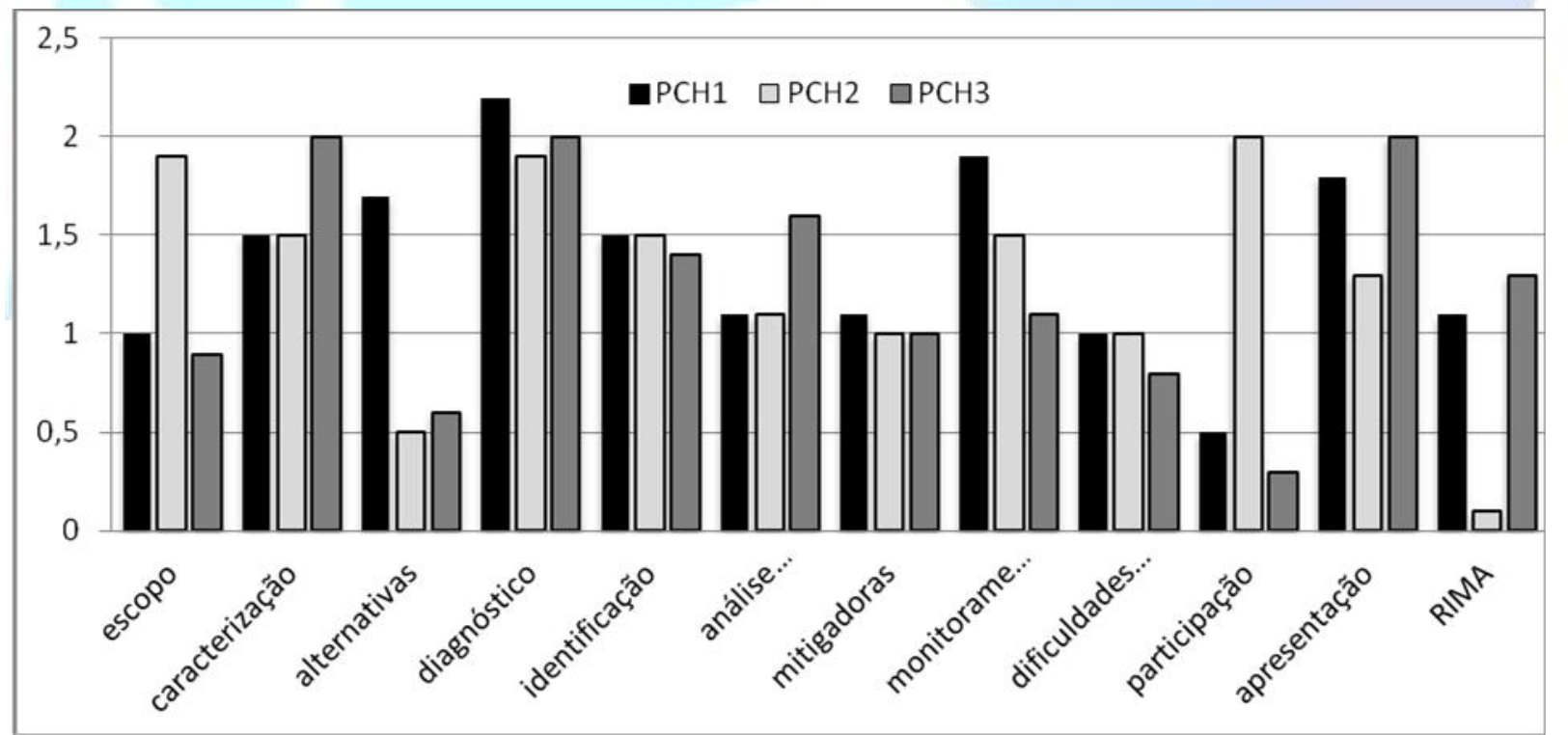

Figura 1. Resultados da ponderação para os critérios empregados na revisão da qualidade dos EIAs para as PCHs 1 a 3. 


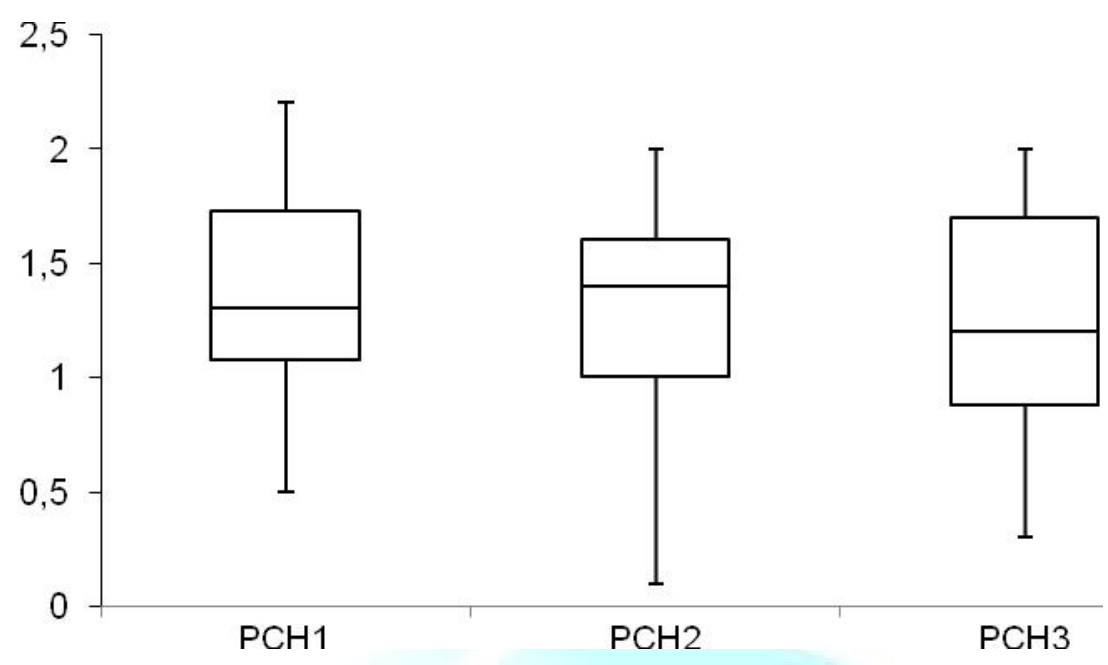

Figura 2. Box-plot para o conjunto de critérios empregados na revisão da qualidade dos EIAs para as PCHs 1 a 3.

Em termos relativos, verifica-se um desempenho regular de todos os EIAs diante dos critérios aplicados. Ainda assim, a metodologia proposta por Pinho et al. (2007) foi útil para a comparação entre os estudos, sendo que a melhor qualidade técnica apresentada pelo EIA da PCH1 foi expressa pela média dos critérios: 1.37 contra 1.28 e 1.25 respectivamente. Este EIA também apresentou melhor desempenho quando se verificam os scores mínimo e máximo atribuídos (mínimo de 0.5 contra 0.1 para a $\mathrm{PCH} 2$ e 0.3 para a $\mathrm{PCH} 3$; e máximo de 2.2 contra 2.0 para as demais). Este comportamento é reforçado pelos valores superiores apresentados para o $1^{\circ}$ e $3^{\circ}$ quartis (1.075 contra 1 e 0.875 ; e 1.725 contra 1.6 e 1.7$)$, e amplitude entre os quartis superior à PCH2 (0.65 contra 0.5 para a PCH2) porém inferior à PCH3 (0.65 contra 0.825$)$. Neste caso, os resultados obtidos desfazem a possibilidade de utilizar apenas a mediana como critério de desempenho para os conjuntos (que, neste caso, seria ligeiramente superior para a $\mathrm{PCH} 2$ ).

Em termos qualitativos, a metodologia para revisão da qualidade dos EIAs é útil para evidenciar quais são os critérios em que o desempenho de um é superior em relação aos demais. Ainda que não tenham sido aplicados pesos entre os critérios, é útil refletir sobre o desempenho pontual apresentado por cada EIA. Neste caso, o documento encaminhado para a PCH1 também apresentou um desempenho superior, tendo alcançado scores mais elevados para os critérios de estudo de alternativas, diagnóstico ambiental, monitoramento, forma de apresentação das informações. Por sua vez, o EIA da $\mathrm{PCH} 2$ apresentou desempenho superior para os critérios definição do escopo, e participação da sociedade. Por fim, o EIA para a PCH3 obteve scores mais elevados para os critérios de caracterização do projeto, descrição dos impactos previstos, apresentação dos resultados e RIMA.

Nos EIAs analisados, foi possível perceber que há um esforço concentrado no diagnóstico ambiental. Nos casos das PCHs 1 e 3, verifica-se que apesar de terem alcançado um bom desempenho na elaboração do diagnóstico ambiental, a identificação dos impactos foi classificada apenas como satisfatório (PCH1) e 'regular' (PCH3), e a descrição dos impactos foi 'regular' para a $\mathrm{PCH} 1$ e 'satisfatório' para a $\mathrm{PCH} 3$.

Esta situação caracteriza o que Sánchez (2006) chamou de 'abordagem exaustiva', que tende a descrever detalhadamente os variados componentes do meio. Normalmente empregada na elaboração de estudos de impacto, tal abordagem causa dificuldades para a integração das informações de diagnóstico à análise dos impactos ambientais e, por vezes, limitando as condições 
para a realização de reflexões e sínteses sobre o estado do meio ambiente por parte da equipe que elabora os estudos.

A qualidade dos estudos analisados deixa muito a desejar em dois pontos importantes para a Avaliação de Impacto Ambiental - escolha da alternativa locacional e participação da sociedade. No caso dos EIAs elaborados para as PCHs 2 e 3, não são consideradas alternativas locacionais para a implantação do empreendimento, que são fundamentais para a discussão da viabilidade ambiental. Segundo a Resolução CONAMA 001/1986 (BRASIL, 1986) devem ser apresentadas em todos os EIAs, devendo ser justificadas a partir de critérios ambientais e não meramente econômicos.

Quanto à participação da sociedade, que não deve se restringir às audiências públicas para discussão dos resultados dos estudos de impacto, verifica-se um desempenho fraco e muito fraco nos casos das PCHs 1 e 3. Pelas informações constantes nos EIAs, não houve qualquer tipo de consulta ou envolvimento com a população durante a elaboração dos estudos, o que inviabiliza a identificação de seus valores e opiniões no momento da avaliação dos impactos.

Em linhas gerais, tendo em vista os resultados obtidos pela aplicação dos critérios de revisão, indica que os EIAs analisados não são suficientes para avaliar a viabilidade ambiental dos empreendimentos, que é o objetivo fundamental do processo de Avaliação de Impacto Ambiental e, portanto, deveriam ser recusados pelo órgão ambiental.

Na prática, tais observações demonstram que as deficiências identificadas nos EIAs para as três PCHs estudadas não são exclusivas da estrutura da AIA no Brasil; ao contrário, como se verifica em trabalhos semelhantes, encontram eco em outros sistemas no mundo.

\section{CONSIDERAÇÕES FINAIS}

A metodologia adotada, representada pela matriz de critérios e subcritérios, inicialmente desenvolvida para a realidade de Portugal, mostrou-se adequada à avaliação da qualidade dos estudos ambientais analisados no âmbito do processo de licenciamento brasileiro. Secundariamente, ao se exercitar a aplicação dos critérios para os respectivos casos, foi possível identificar as deficiências técnicas apresentadas por todos os EIAs/RIMAs; o método, da mesma forma, possibilitou comparar a qualidade dos EIAs estudados entre si.

Uma vez que o EIA é um dos elementos (muitas vezes, o único) que subsidiam a decisão de conceder ou não as devidas licenças, os resultados de sua análise, se conduzidos por meio de uma matriz de critérios como a que foi aplicada no presente trabalho, podem auxiliar mais claramente os tomadores de decisão, diminuindo, assim, seu grau de subjetividade.

Considerando que os três estudos não apresentaram desempenho satisfatório em função do conjunto de critérios aplicados, prejudicando a análise de viabilidade ambiental dos empreendimentos propostos, torna-se premente a necessidade dos órgãos licenciadores reavaliarem o processo de licenciamento ambiental para PCHs, face aos impactos provocados por este tipo de empreendimento. Adicionalmente, é preciso incorporar ao processo de licenciamento a devida avaliação dos impactos cumulativos e sinérgicos, bem como daqueles oriundos da etapa de desativação dessas centrais.

Finalmente, tendo em a variabilidade no desempenho dos estudos de impactos, conforme discutidas ao longo deste trabalho entende-se, portanto, que a aplicação de procedimentos sistemáticos para análise de estudos ambientais pode oferecer aos técnicos envolvidos condições mais favoráveis para a organização e interpretação das informações apresentadas, o que teria um efeito benéfico sobre o processo de AIA como um todo, bem como sobre a qualidade de seus pareceres. 


\section{REFERÊNCIAS}

BANCO MUNDIAL. Licenciamento ambiental de empreendimentos hidrelétricos no Brasil: uma contribuição para o debate. Relatório 40995/BR. Brasília, 2008. 34 p.

BARBOSA, T.A.S.; DUPAS, F.A. Matriz simplificada para avaliar impactos ambientais em Pequenas Centrais Hidrelétricas. Revista Brasileira de Energia, Itajubá, v. 12 n. 2, pg. 1-9, 2006.

BARKER, A.; WOOD, C. An evaluation of EIA system performance in eight EU countries. Environmental Impact Assessment Review, New York, v.19, p.387-404, 1999.

BRASIL. Leis, etc. Resolução CONAMA 001 de 06 de janeiro de 1986. Dispõe sobre a regulamentação da AIA no país. Conselho Nacional do Meio Ambiente, Brasília, 1986.

CANELAS, L.; ALMANSA, P.; MERCHAN, M.; CIFUENTES, P. Quality of environmental impact statements in Portugal and Spain. Environmental Impact Assessment Review, New York, v.25, p.217-225, 2005.

CERPCH - Centro nacional de referência em pequenas centrais hidrelétricas. Qual a perspectiva das PCHs para o futuro? PCH Notícia, Itajubá, Ano 8, n 31, p. 40-47, 2006.

EUROPEAN COMISSION. Guidance on EIA - EIS Review. Directorate General for the Environment. Luxembourg, 2001. 29p.

GLASSON, J.; THERIVEL, R.; CHADWICK, A. Introduction to Environmental Impact Assessment. 3rd Edition, New York: Routledge, 2005. 496p.

GLASSON, J.; SALVADOR, N.N.B. EIA in Brazil: a procedures-practice gap. A comparative study with reference to the European Union, and especially the UK. Environmental Impact Assessment Review, New York, v.20, p.191-225, 2000.

HICKIE, D.; WADE, M. Development of guidelines for improving the effectiveness of environmental assessment. Environmental Impact Assessment Review, New York, v.18, p.267287, 1998.

LEE, N.; COLLEY, R. Reviewing the quality of environmental statements: review methods and findings. Town Plan Rev, Liverpool, 62(2), pg.239-248. 1992.

MINISTÉRIO DE MINAS E ENERGIA. Programa de Incentivo às Fontes Alternativas de Energia Elétrica. http://www.mme.gov.br/programs_display.do?chn=877. 2009.

MONTAÑO, M.; SOUZA, M.P. A viabilidade ambiental no licenciamento de empreendimentos perigosos no Estado de São Paulo. Eng. Sanit. Ambient., Rio de Janeiro, v.13, n.4, p.435-442, 2008.

MINISTÉRIO PÚBLICO DA UNIÃO. Deficiências em estudos de impacto ambiental. Ministério Público Federal, $4^{\mathrm{a}}$ Câmara de Coordenação e Revisão: Escola Superior do Ministério Público da União, Brasília, 2004. 38p. 
NASCIMENTO, E. P.; DRUMMOND, J.A. Invenção e realidade da região de Belo Monte. In: (org.). Amazônia - Dinamismo econômico e conservação ambiental. Rio de

Janeiro: Garamond, p. 35-57, 2003.

PETERSON, K. Quality of environmental impact statements and variability of scrutiny by reviewers. Environmental Impact Assessment Review, New York, v.30, p.169-176, 2010.

PINHO, P.; MAIA, R.; MONTERROSO, A. The quality of Portuguese Environmental Impact Studies: the case of small hydropower projects. Environmental Impact Assessment Review, New York, v. 27, p. 189-205, 2007.

SÁNCHEZ, L.E. Avaliação de Impacto Ambiental: conceitos e métodos. São Paulo: Oficina de Textos, 2006. 495p.

THIAGO FILHO, G.L.; VIANA, A.N.; GUEDES, A.C. Pequenas Centrais Hidrelétricas. In: TOLMASQUIM, M. T. (org.). Fontes Renováveis de Energia no Brasil. Rio de Janeiro: Relumé Dumará, p. 163-206, 2004.

TSOUTSOS, T.; MARIA, E.; MATHIOUDAKIS, V. Sustainable siting procedure of small hydroelectric plants: the Greek experience. Energy Policy, Philadelphia, n.35, p.2946-2959, 2007. 